Original article

\title{
Socioeconomic Trends in Adolescent Smoking in Finland From 2000 to 2015
}

\author{
Noora Knaappila ${ }^{\text {a,* }}$, Mauri Marttunen, M.D., Ph.D. ${ }^{\text {, }}$ Sari Fröjd, Ph.D. ${ }^{\text {a }}$, \\ Nina Lindberg, M.D., Ph.D. ${ }^{\text {, }}$, and Riittakerttu Kaltiala-Heino, M.D., Ph.D. ${ }^{\text {a, d,e }}$ \\ ${ }^{\text {a }}$ Faculty of Medicine and Life Sciences, University of Tampere, Tampere, Finland \\ ${ }^{\mathrm{b}}$ University of Helsinki and Helsinki University Hospital, Adolescent Psychiatry, Helsinki Finland \\ ${ }^{\mathrm{c}}$ Helsinki University Central Hospital, Department of Adolescent Psychiatry, HUS, Helsinki, Finland \\ ${ }^{\mathrm{d}}$ Vanha Vaasa Hospital, Vaasa, Finland \\ e Tampere University Hospital, Department of Adolescent Psychiatry, Pitkäniemi, Finland
}

Article history: Received August 29, 2018; Accepted November 13, 2018

Keywords: Smoking; Adolescent; Epidemiology; Surveys and questionnaires; Databases; Factual; Population surveillance; Socioeconomic factors

\section{A B S T R A C T}

Purpose: Smoking is a significant cause of morbidity and mortality worldwide. In the past decades, the prevalence of adolescent smoking has decreased in industrial countries. However, whether the decreasing trend can be seen across all socioeconomic groups is unknown. The aim of this study was to examine time trends in adolescent smoking according to the socioeconomic status among Finnish adolescents between 2000 and 2015.

Methods: A population-based school survey was conducted biennially among 14- to 16-year-old Finns between 2000 and 2015 ( $\mathrm{n}=761,278$ ). Distributions for frequent smoking, lifelong nonsmoking, and socioeconomic adversities (low parental education, not living with both parents and parental unemployment during the past year) were calculated. Associations were studied using binomial logistic regression results shown by odds ratios with $95 \%$ confidence intervals.

Results: Frequent smoking was positively associated and lifelong nonsmoking was negatively associated with socioeconomic adversities. Over the study period, the overall prevalence of frequent smoking decreased and lifelong nonsmoking increased. However, no similar changes were observed among adolescents with most socioeconomic adversities.

Conclusion: Socioeconomic differences in adolescent smoking increased in Finland between 2000 and 2015. Although the overall prevalence of frequent smoking decreased, no similar decrease was observed among adolescents with most socioeconomic adversities. Similarly, although the overall prevalence of lifelong nonsmoking increased, this was not observed among adolescents with most socioeconomic adversities. Socioeconomic adversities should be considered in the prevention of adolescent smoking.

$$
\text { (c) } 2018 \text { Society for Adolescent Health and Medicine. All rights reserved. }
$$

\section{IMPLICATIONS AND CONTRIBUTIONS}

Socioeconomic differences in adolescent smoking increased among Finnish adolescents between 2000 and 2015. Although the overall prevalence of frequent smoking decreased and lifelong nonsmoking increased, no similar changes were observed among adolescents with most socioeconomic adversities. Socioecononomic adversities should be considered in the prevention of adolescent smoking.
Smoking is a significant cause of morbidity and mortality worldwide. It is a major risk factor of cancer and cardiovascular diseases, which are leading causes of death in the industrial countries [1,2]. In Europe, approximately $12 \%$ of adolescent boys and $11 \%$ of girls smoke at least once a week, although the prevalences vary largely between countries [3]. Over the past

Conflicts of interest: The authors have no conflicts of interest to disclose.

* Address correspondence to: Noora Knaappila, Faculty of Medicine and Life Sciences, University of Tampere, 33014 Tampere, Finland.

E-mail address: knaappila.noora.x@student.uta.fi (N. Knaappila). 
Table 1

Lifelong nonsmoking, frequent smoking, and socioeconomic characteristics among Finnish boys and girls in the eighth and ninth grades of comprehensive school

\begin{tabular}{llll} 
& $\begin{array}{l}\text { Boys } \\
(\mathrm{n}=381,527)\end{array}$ & $\begin{array}{l}\text { Girls } \\
(\mathrm{n}=376,814)\end{array}$ & $p$ \\
\hline Age, mean (SD) & $15.4(.7)$ & $15.3(.6)$ & $<.001$ \\
Lifelong nonsmoking (\%) & & & $<.001$ \\
$\quad$ Yes & 53.6 & 55.5 & \\
No & 44.8 & 43.4 & \\
Missing & 1.7 & 1.1 & $<.001$ \\
Frequent smoking (\%) & & & \\
Yes & 21.7 & 20.0 & \\
No & 76.7 & 78.9 & \\
Missing & 1.7 & 1.1 & \\
Lives with both parents (\%) & & & \\
Yes & 74.4 & 73.7 & \\
No & 23.3 & 25.1 & \\
Missing & 2.3 & 1.2 & \\
Both parents only basic education & & & \\
(\%) & & & \\
Yes & 5.6 & 5.9 & \\
No & 86.8 & 87.5 & \\
Missing & 7.6 & 6.6 & \\
Parental unemployment past year & & & \\
(\%) & 70.9 & 69.9 & \\
No & 23.6 & 25.6 & \\
One parent & 3.2 & 3.3 & \\
Both parents & 2.3 & & \\
Missing & & & \\
\hline
\end{tabular}

$\mathrm{SD}=$ standard deviation

decades, the prevalence of adolescent smoking has decreased in Western countries, including Finland [3-9]. Finland has been one of the world's pioneer countries in reducing smoking since 1977, when the Tobacco Act came into force [10]. The objective of the Tobacco Act is to end the use of tobacco and other nicotine products in Finland by 2030 [11]. The main areas and measures for implementing tobacco policy in Finland are health education, price policy, restrictions, research and development [10].

Smoking prevention requires scientific knowledge on the risk factors of smoking. Several risk factors of adolescent smoking have been identified in the scientific literature, including male gender [3,12], parental smoking [13], genetic factors [14], negative life events [14], mental health problems [15], and smoking peers [15]. In addition, indicators of socioeconomic status (SES) have been examined as risk factors of adolescent smoking. SES is an aggregate concept comprising resource-based (such as material and social resources) and prestige-based (individual's rank or status) indicators of socioeconomic position, which can be measured at individual, household, and neighborhood levels [16]. It can be assessed not only through individual measures, such as education, income, or occupation, but also through composite measures that provide an overall index of socioeconomic level [17].

Of the SES indicators, low parental education has been associated with adolescent smoking $[4,7,8]$. The likelihood of smoking has also been observed to be more common among adolescents not living with both parents than among those living in intact families [18-20]. However, the association between SES and smoking varies over time and between countries. According to the diffusion of innovations theory by Rogers [21] and the smoking epidemic model by Lopez et al. [22], smoking starts first in higher socioeconomic groups (stage I), and the rest of the population follows later (stage II). As knowledge of the health hazards of smoking increases, smoking starts to level off (stage III) and finally decrease (stage IV), which also occurs first in higher socioeconomic groups, and other groups follow later. Many European countries, including Finland, seem to have reached the fourth stage of the smoking epidemic in the 21st century [23].

Although the overall level of adolescent smoking has decreased, scientific evidence suggests that socioeconomic differences in adolescent smoking may have increased in Western countries in the 21 st century. Socioeconomic health disparities can be measured both through absolute measures, such as risk differences, and relative measures, such as risk ratios [24]. Absolute deprivation theory suggests that differential health outcomes result primarily from exposure to socioeconomic adversities, such as poverty, low education, and limited health services, whereas relative deprivation theory suggests that relative deprivation, embodied by psychosocial stress, leads to health disparities by influencing an individual's sense of wellbeing and subsequent health behaviors [24]. Both absolute and relative measures are used in the scientific literature, and both are meaningful measures for monitoring inequality. In a European time trend study [25], absolute educational differences in adolescent smoking increased in Croatia and Italy, and relative educational differences in adolescent smoking increased especially in the Netherlands and Belgium between 2002 and 2010. Richter and Leppin [26] observed that the level of socioeconomic disparities in adolescent smoking remained virtually unchanged in Germany between 1994 and 2002. Rasmussen et al. [9] found that socioeconomic differences in adolescent smoking fluctuated between 1991 and 2006. In addition, one study on the subject was conducted in Finland between 1977 and 2007 [27], in which absolute differences in adolescent smoking according to parental education level increased. Decreasing socioeconomic health disparities is an important public health objective, and therefore, data on such disparities is required [28]. The aim of this study was to examine socioeconomic differences in smoking among Finnish adolescents between 2000 and 2015. Our research questions include the following:

(1) Did the prevalences of frequent smoking and lifelong nonsmoking change among Finnish adolescents between years 2000 and 2015?

(2) Are frequent smoking and lifelong nonsmoking associated with socioeconomic adversities (low parental education, not living with both parents and parental unemployment) among Finnish adolescents?

(3) Did the differences between socioeconomic groups in frequent smoking and lifelong nonsmoking increase or decrease among Finnish adolescents between years 2000 and 2015?

\section{Methods}

\section{Data and participants}

The School Health Promotion Study by the National Institute for Health and Welfare is a survey that examines the health, health behavior, and school experiences of Finnish adolescents. The survey has been conducted biennially since 1996 among eighth and ninth graders with pooled 2-year data (2000-2001, 2002-2003, 2004-2005, 2006-2007, 2008-2009, 2010-2011, 
Table 2

Lifelong nonsmoking and frequent smoking over time among Finnish boys and girls in the eighth and ninth grades of comprehensive school ${ }^{\mathrm{a}}$

\begin{tabular}{|c|c|c|c|c|c|c|c|}
\hline & $2002-2003$ & 2004-2005 & $2006-2007$ & $2008-2009$ & $2010-2011$ & $2012-2013$ & 2014-2015 \\
\hline \multicolumn{8}{|l|}{ Boys } \\
\hline Lifelong nonsmoking & $1.2(1.2-1.3)$ & $1.6(1.5-1.6)$ & $1.8(1.8-1.9)$ & $1.8(1.7-1.8)$ & $1.9(1.8-1.9)$ & $2.0(1.9-2.0)$ & $3.2(3.0-3.3)$ \\
\hline Frequent smoking & $.8(.8-.8)$ & $.7(.6-.7)$ & $.6(.6-.6)$ & $.6(.6-.6)$ & $.6(.6-.6)$ & $.5(.5-.6)$ & $.4(.3-.4)$ \\
\hline \multicolumn{8}{|l|}{ Girls } \\
\hline Lifelong nonsmoking & $1.3(1.2-1.3)$ & $1.6(1.5-1.6)$ & $1.8(1.7-1.8)$ & $1.9(1.8-1.9)$ & $1.9(1.8-1.9)$ & $2.3(2.3-2.4)$ & $3.8(3.6-3.9)$ \\
\hline Frequent smoking & $.8(.8-.9)$ & $.7(.7-.7)$ & $.6(.5-.6)$ & $.6(.5-.6)$ & $.6(.5-.6)$ & $.5(.4-.5)$ & $.3(.3-.3)$ \\
\hline
\end{tabular}

Values are presented as OR $(95 \% \mathrm{CI})$.

$\mathrm{CI}=$ confidence interval; $\mathrm{OR}=$ odds ratio.

a Time period 2000-2001 is used as a reference category.

2012-2013, 2014-2015). The data were collected anonymously during a school lesson under the supervision of a teacher, who did not interfere with the responses. Participants were informed about the voluntary nature of the study in both orally and in writing, and returning the questionnaire was taken to be consent to participate. The questionnaire took about 30-45 minutes to complete. After this, the questionnaires were put in an envelope, sealed, and returned directly to the research center. The timing of the study, sampling, and data collection methods were held constant in each survey round. Altogether, 761,278 (50,404109,127 biennially) eighth and ninth graders participated in the survey. The eighth graders were aged 14-15 years and the ninth graders 15-16 years at the time of the surveys. When the nonresponders were excluded, the biennial cohorts covered between $43 \%$ and $82 \%$ of the whole age cohort of the country. The study was approved by the ethics committee of Pirkanmaa Hospital District and the National Institute of Health and Welfare.

\section{Measures}

Smoking was elicited with two questions, the first one measuring lifelong smoking: "How many cigarettes, pipefuls and cigars have you smoked altogether?" The response alternatives

Table 3

Lifelong nonsmoking and frequent smoking by socioeconomic adversities among Finnish boys and girls in the eighth and ninth grades of comprehensive school

\begin{tabular}{|c|c|c|}
\hline & \multicolumn{2}{|c|}{ Lifelong nonsmoking Frequent smoking } \\
\hline \multicolumn{3}{|l|}{ Boys } \\
\hline \multicolumn{3}{|l|}{ Family structure } \\
\hline Both parents & ref & ref \\
\hline Not living with both parents & $.6(.6-.6)$ & $2.1(2.0-2.1)$ \\
\hline \multicolumn{3}{|c|}{ Both parents with low education } \\
\hline No & ref & ref \\
\hline Yes & $.7(.7-.7)$ & $1.7(1.6-1.7)$ \\
\hline \multicolumn{3}{|l|}{ Parental unemployment } \\
\hline Neither parent & ref & ref \\
\hline One parent & $.8(.8-.8)$ & $1.4(1.4-1.5)$ \\
\hline Both parents & $.5(.5-.5)$ & $2.6(2.5-2.7)$ \\
\hline \multicolumn{3}{|l|}{ Girls } \\
\hline \multicolumn{3}{|l|}{ Family structure } \\
\hline Both parents & ref & ref \\
\hline Not living with both parents & $.5(.5-.5)$ & $2.2(2.1-2.2)$ \\
\hline \multicolumn{3}{|c|}{ Both parents with low education } \\
\hline No & ref & ref \\
\hline Yes & $.7(.7-.8)$ & $1.6(1.5-1.6)$ \\
\hline \multicolumn{3}{|l|}{ Parental unemployment } \\
\hline Neither parent & ref & ref \\
\hline One parent & $.7(.7-.7)$ & $1.5(1.5-1.6)$ \\
\hline Both parents & $.6(.5-.6)$ & $2.3(2.2-2.4)$ \\
\hline
\end{tabular}

Values are presented as OR $(95 \% \mathrm{CI})$.

$\mathrm{CI}=$ confidence interval; $\mathrm{OR}=$ odds ratio. were "none/only one/about $2-50 / 50$ or more." The second question measured current smoking: "Which of the following alternatives describes best your CURRENT SMOKING?" The response alternatives were "I smoke once a day or more often/I smoke once a week or more often but not daily/I smoke less often than once a week/I have quit smoking." For the analyses, two dichotomous variables were created: "frequent smoking," in which smoking once a week or more often was regarded as frequent smoking, and "lifelong nonsmoking," which was dichotomized as having never tried smoking versus having tried smoking at least once.

The socioeconomic variables recorded were parental education, parental unemployment during the past year, and family structure. Parental education was elicited as follows: "What is the highest educational qualification your father/mother has achieved?" The response options in the 2000 questionnaire were "basic school/vocational school/high school and/or vocational school/university or polytechnic." The response options varied a little over time: for instance, in the 2013 questionnaire, there was a response option "no education," which was removed again in the 2015 questionnaire. For the analyses, parental education was dichotomized to parental basic education only (including the response alternative "no education") versus other. Parental unemployment was elicited as follows: "Have your parents been unemployed or laid off work during the past YEAR?" The response alternatives were the same in all questionnaires: "neither/one parent/both parents." The family structure was elicited as follows: "My family consists of..." The response options in the 2000 questionnaire were: "mother and father/ mother and stepfather/father and stepmother/mother only/father only/spouse/other caregiver." The response options varied slightly over time. For the analyses, family structure was dichotomized to living with both parents versus other. In this article, all three variables are referred to as socioeconomic adversities. In addition, a variable "cumulative socioeconomic adversity" was created, in which all three socioeconomic variables were combined: a score of 0 stood for having no socioeconomic adversities studied (living with both parents, no parental unemployment and at least one parent with higher than basic education) and a score of 4 stood for having all socioeconomic adversities studied (not living with both parents, both parents unemployed, and both parents with basic education only). The prevalences of socioeconomic adversities are presented elsewhere [29].

\section{Statistical analysis}

All statistical analyses were conducted using SPSS software (version 24). Bivariate associations were studied using binomial 


\section{Table 4}

Frequent smoking and lifelong nonsmoking over time by cumulative socioeconomic adversity among Finnish boys and girls in the eighth and ninth grades of comprehensive school

\begin{tabular}{|c|c|c|c|c|c|c|c|c|}
\hline $\begin{array}{l}\text { Number of } \\
\text { sociodemographic } \\
\text { adversities }\end{array}$ & $2000-2001$ & $2002-2003$ & 2004-2005 & 2006-2007 & 2008-2009 & 2010-2011 & 2012-2013 & 2014-2015 \\
\hline \multicolumn{9}{|c|}{ Frequent smoking boys } \\
\hline 0 & $24.3(4,924 / 20,280)$ & $20.7(5,546 / 26,737)$ & $16.5(4,683 / 28,427)$ & $15.1(4,611 / 30,572)$ & $16.2(4,767 / 29,479)$ & $15.6(4,120 / 26,408)$ & $12.9(2,900 / 22,457)$ & $8.6(972 / 11,238)$ \\
\hline 1 & $31.1(3,964 / 12,728)$ & $28.8(4,251 / 14,775)$ & $25.2(3,827 / 15,212)$ & $22.8(3,332 / 14,605)$ & $24.0(3,575 / 14,917)$ & $22.6(3,437 / 15,241)$ & $19.7(2,960 / 15,016)$ & $13.3(1,090 / 8,195)$ \\
\hline 2 & $40.8(1,916 / 4,696)$ & $38.2(1,913 / 5,005)$ & $32.2(1,567 / 4,874)$ & $31.6(1,415 / 4,478)$ & $31.3(1,461 / 4,664)$ & $31.3(1,589 / 5,076)$ & $28.4(1,559 / 5,495)$ & $18.6(586 / 3,158)$ \\
\hline 3 & $46.5(418 / 898)$ & $43.4(371 / 854)$ & $42.7(367 / 859)$ & $42.3(302 / 714)$ & $44.3(295 / 666)$ & $42.6(377 / 886)$ & $37.8(335 / 886)$ & $27.9(150 / 538)$ \\
\hline 4 & $67.3(74 / 110)$ & $63.8(83 / 130)$ & $58.8(70 / 119)$ & $72.7(96 / 132)$ & $74.1(106 / 143)$ & $77.3(136 / 176)$ & $63.8(143 / 224)$ & $64.5(127 / 197)$ \\
\hline \multicolumn{9}{|c|}{ Frequent smoking girls } \\
\hline 0 & $22.2(4,287 / 19,334)$ & $19.3(4,807 / 24,767)$ & $16.3(4,373 / 26,882)$ & $13.2(3,895 / 29,410)$ & $13.5(3,871 / 28,625)$ & $13.1(3,341 / 25,437)$ & $10.0(2,189 / 21,970)$ & $6.3(712 / 11,269)$ \\
\hline 1 & $30.4(3,875 / 12,767)$ & $27.7(4,027 / 14,563)$ & $24.4(3,707 / 15,168)$ & $21.4(3,304 / 15,445)$ & $21.2(3,358 / 15,846)$ & $20.3(3,178 / 15,644)$ & $17.0(2,599 / 15,316)$ & $11.2(958 / 8,577)$ \\
\hline 2 & $39.3(2,039 / 5,188)$ & $37.6(2,017 / 5,360)$ & $34.1(1,930 / 5,662)$ & $30.8(1,557 / 5,062)$ & $29.9(1,518 / 5,085)$ & $29.2(1,691 / 5,785)$ & $24.3(1,502 / 6,170)$ & $17.5(624 / 3,556)$ \\
\hline 3 & $43.6(422 / 968)$ & $41.1(395 / 960)$ & $41.9(371 / 885)$ & $37.4(280 / 749)$ & $38.0(290 / 764)$ & $36.9(398 / 1,078)$ & $30.1(321 / 1,068)$ & $25.6(168 / 656)$ \\
\hline 4 & $52.9(45 / 85)$ & $57.0(53 / 93)$ & $61.8(55 / 89)$ & $58.2(57 / 98)$ & $63.7(65 / 102)$ & $65.0(106 / 163)$ & $59.3(102 / 172)$ & $55.4(51 / 92)$ \\
\hline \multicolumn{9}{|c|}{ Lifelong nonsmoking boys } \\
\hline 0 & $45.4(9,209 / 20,280)$ & $51.3(13,708 / 26,737)$ & $57.8(16,431 / 28,427)$ & $60.7(18,559 / 30,572)$ & $60.1(17,708 / 29,479)$ & $62.0(16,370 / 26,408)$ & $64.1(14,404 / 22,457)$ & $74.2(8,338 / 11,238)$ \\
\hline 1 & $39.7(5,052 / 12,728)$ & $43.8(6,465 / 14,775)$ & $48.8(7,423 / 15,212)$ & $52.2(7,617 / 14,605)$ & $51.8(7,730 / 14,917)$ & $54.0(8,237 / 15,241)$ & $56.3(8,457 / 15,016)$ & $66.9(5,479 / 8,195)$ \\
\hline 2 & $32.7(1,535 / 4,696)$ & $35.5(1,778 / 5,005)$ & $41.6(2,027 / 4,874)$ & $43.0(1,924 / 4,478)$ & $44.2(2,060 / 4,664)$ & $45.0(2,283 / 5,076)$ & $48.0(2,639 / 5,495)$ & $60.2(1,902 / 3,158)$ \\
\hline 3 & $30.1(270 / 898)$ & $30.3(259 / 854)$ & $33.4(287 / 859)$ & $32.3(230 / 714)$ & $34.5(230 / 666)$ & $34.1(302 / 886)$ & $39.6(351 / 886)$ & $50.9(274 / 538)$ \\
\hline 4 & $20.9(23 / 110)$ & $21.5(28 / 130)$ & $19.3(23 / 119)$ & $13.6(18 / 132)$ & $11.2(16 / 143)$ & $13.6(24 / 176)$ & $21.4(48 / 224)$ & $23.4(46 / 197)$ \\
\hline \multicolumn{9}{|c|}{ Lifelong nonsmoking girls } \\
\hline 0 & $46.9(9,066 / 19,334)$ & $53.7(13,392 / 24,946)$ & $59.3(15,949 / 26,882)$ & $62.2(18,301 / 29,410)$ & $63.1(18,063 / 28,625)$ & $64.6(16,436 / 25,437)$ & $70.5(15,499 / 21,970)$ & $79.8(8,989 / 11,269)$ \\
\hline 1 & $40.7(5,193 / 12,767)$ & $44.5(6,478 / 14,563)$ & $49.1(7,442 / 15,168)$ & $52.2(8,057 / 15,445)$ & $53.0(8,404 / 15,846)$ & $54.5(8,532 / 15,644)$ & $60.5(9,267 / 15,316)$ & $71.4(6,122 / 8,577)$ \\
\hline 2 & $33.8(1,755 / 5,188)$ & $35.7(1,913 / 5,360)$ & $41.1(2,327 / 5,662)$ & $41.2(2,085 / 5,062)$ & $44.1(2,241 / 5,085)$ & $44.9(2,597 / 5,785)$ & $50.8(3,136 / 6,170)$ & $62.1(2,208 / 3,556)$ \\
\hline 3 & $30.8(298 / 968)$ & $31.7(304 / 960)$ & $33.9(300 / 885)$ & $36.6(274 / 749)$ & $38.2(292 / 764)$ & $40.1(432 / 1,078)$ & $45.0(481 / 1,068)$ & $53.2(349 / 656)$ \\
\hline 4 & $23.5(20 / 85)$ & $24.7(23 / 93)$ & $24.7(22 / 89)$ & $21.4(21 / 98)$ & $21.6(22 / 102)$ & $23.3(38 / 163)$ & $24.4(42 / 172)$ & $25.0(23 / 92)$ \\
\hline
\end{tabular}

Values are presented as $\%(\mathrm{n} / \mathrm{N})$. 
logistic regression results shown as odds ratios with 95\% confidence intervals. Frequent smoking and lifelong nonsmoking were entered as dependent variables. In the first model, categorical time periods (2000-2001, 2002-2003, 2004-2005, 2006-2007, 2008-2009, 2010-2011, 2012-2013, 2014-2015) were entered as independent factors, using the time period 2000-2001 as a reference category. In the second model, family structure (living with both parents/other), parental unemployment during the past year (neither/one parent/both parents), and parental education (both parents basic education only/other) were entered as independent factors one at a time. In the third model, the file was split according to categorical time periods, and cumulative socioeconomic adversity was entered as an independent factor.

\section{Results}

The overall prevalence of frequent smoking was $22 \%$ among boys and 20\% among girls. Fifty-four percent of boys and 54\% of girls had never tried smoking (Table 1). At the overall level, the ORs for frequent smoking decreased among both sexes over the study period, whereas the ORs for lifelong nonsmoking increased among both sexes over time (Table 2).

Socioeconomic differences were observed both in frequent smoking and lifelong nonsmoking (Table 3). Frequent smoking was more common among boys and girls not living with both parents than among those living with both parents. Frequent smoking was more common among boys and girls both of whose parents had only basic education than among those who had at least one parent with higher than basic education. Frequent smoking was also positively associated with parental unemployment during the past year among both sexes. Opposite associations were observed in lifelong nonsmoking.
The prevalences of smoking according to cumulative socioeconomic adversity are presented in Table 4 . The prevalence of frequent smoking decreased among boys and girls with the least socioeconomic adversities over the study period, whereas no decrease was observed among adolescents with most socioeconomic adversities. Similarly, the prevalence of lifelong nonsmoking increased among adolescents with least socioeconomic adversities, whereas they varied only slightly among those with most socioeconomic adversities (Tables 4 and 5). The relative differences according to cumulative socioeconomic adversity are presented in Table 5 . The ORs in frequent smoking between adolescents not living with both parents, with both parents unemployed, and with parents having basic education only and adolescents living with both parents, with no parental unemployment, and at least one parent with higher than basic education increased among both sexes over the study period. The ORs in lifelong nonsmoking according to cumulative socioeconomic adversity varied only slightly over time.

\section{Discussion}

In this study, both frequent smoking and lifelong nonsmoking were associated with socioeconomic adversities among 14- to 16-year-old adolescents in Finland. The prevalence of frequent smoking was greater among adolescents with any of the socioeconomic adversities studied than among those with no socioeconomic adversities. Conversely, the prevalence of lifelong nonsmoking was lower among adolescents with any of the socioeconomic adversities studied than among those with no socioeconomic adversities. Frequent smoking was positively associated and lifelong nonsmoking was negatively associated with the number of socioeconomic adversities. Most importantly, although the overall prevalences of frequent smoking decreased and lifelong nonsmoking increased, no similar

\section{Table 5}

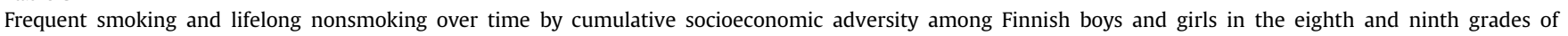
comprehensive school $^{\mathrm{a}}$

\begin{tabular}{|c|c|c|c|c|c|c|c|c|}
\hline $\begin{array}{l}\text { Number of } \\
\text { sociodemographic } \\
\text { adversities }\end{array}$ & $2000-2001$ & $2002-2003$ & 2004-2005 & $2006-2007$ & 2008-2009 & 2010-2011 & $2012-2013$ & $2014-2015$ \\
\hline \multicolumn{9}{|c|}{ Frequent smoking boys } \\
\hline 1 & $1.4(1.3-1.5)$ & $1.5(1.5-1.6)$ & $1.7(1.6-1.8)$ & $1.7(1.6-1.8)$ & $1.6(1.6-1.7)$ & $1.6(1.5-1.7)$ & $1.7(1.6-1.8)$ & $1.6(1.5-1.8)$ \\
\hline 2 & $2.2(2.0-2.3)$ & $2.4(2.2-2.5)$ & $2.4(2.3-2.6)$ & $2.6(2.4-2.8)$ & $2.4(2.2-2.5)$ & $2.5(2.3-2.6)$ & $2.7(2.5-2.9)$ & $2.4(2.2-2.7)$ \\
\hline 3 & $2.7(2.4-3.1)$ & $2.9(2.5-3.4)$ & $3.8(3.3-4.3)$ & $4.2(3.6-4.8)$ & $4.2(3.6-4.9)$ & $4.1(3.6-4.7)$ & $4.1(3.6-4.8)$ & $4.2(3.5-5.2)$ \\
\hline 4 & $6.7(4.4-10.0)$ & $6.7(4.7-9.5)$ & $7.1(4.9-10.3)$ & $15.6(10.5-23.1)$ & $16.3(11.0-24.2)$ & $18.1(12.7-25.9)$ & $13.1(9.9-17.5)$ & $22.8(16.6-31.4)$ \\
\hline \multicolumn{9}{|c|}{ Frequent smoking girls } \\
\hline 1 & $1.5(1.5-1.6)$ & $1.6(1.5-1.7)$ & $1.7(1.6-1.8)$ & $1.8(1.7-1.9)$ & $1.7(1.6-1.8)$ & $1.7(1.6-1.8)$ & $1.8(1.7-2.0)$ & $1.9(1.7-2.1)$ \\
\hline 2 & $2.3(2.1-2.4)$ & $2.5(2.4-2.7)$ & $2.7(2.5-2.8)$ & $2.9(2.7-3.1)$ & $2.7(2.5-2.9)$ & $2.7(2.6-2.9)$ & $2.9(2.7-3.1)$ & $3.2(2.8-3.6)$ \\
\hline 3 & $2.7(2.4-3.1)$ & $2.9(2.6-3.4)$ & $3.7(3.2-4.2)$ & $4.0(3.4-4.6)$ & $3.9(3.4-4.5)$ & $3.9(3.4-4.4)$ & $3.9(3.4-4.5)$ & $5.2(4.3-6.2)$ \\
\hline 4 & $3.9(2.5-6.0)$ & $5.5(3.6-8.3)$ & $8.2(5.3-12.6)$ & $9.4(6.3-14.2)$ & $11.4(7.6-17.1)$ & $12.2(8.8-16.9)$ & $13.2(9.7-18.0)$ & $19.2(12.6-29.4)$ \\
\hline \multicolumn{9}{|c|}{ Lifelong nonsmoking boys } \\
\hline 1 & $.8(.8-.8)$ & $.7(.7-.8)$ & $.7(.7-.7)$ & $.7(.7-.7)$ & $.7(.7-.7)$ & $.7(.7-.7)$ & $.7(.7-.7)$ & $.7(.7-.8)$ \\
\hline 2 & $.6(.5-.6)$ & $.5(.5-.6)$ & $.5(.5-.5)$ & $.5(.5-.5)$ & $.5(.5-.6)$ & $.5(.5-.5)$ & $.5(.5-.5)$ & $.5(.5-.6)$ \\
\hline 3 & $.5(.4-.6)$ & $.4(.4-.5)$ & $.4(.3-.4)$ & $.3(.3-.4)$ & $.3(.3-.4)$ & $.3(.3-.4)$ & $.4(.3-.4)$ & $.4(.3-.4)$ \\
\hline 4 & $.3(.2-.5)$ & $.3(.2-.4)$ & $.2(.1-.3)$ & $.1(.1-.2)$ & $.1(.0-.1)$ & $.1(.1-.1)$ & $.2(.1-.2)$ & $.1(.1-.2)$ \\
\hline \multicolumn{9}{|c|}{ Lifelong nonsmoking girls } \\
\hline 1 & $.8(.7-.8)$ & $.7(.7-.7)$ & $.7(.6-.7)$ & $.7(.6-.7)$ & $.7(.6-.7)$ & $.7(.6-.7)$ & $.6(.6-.7)$ & $.6(.6-.7)$ \\
\hline 2 & $.6(.5-.6)$ & $.5(.4-.5)$ & $.5(.4-.5)$ & $.4(.4-.4)$ & $.5(.4-.5)$ & $.4(.4-.5)$ & $.4(.4-.5)$ & $.4(.4-.4)$ \\
\hline 3 & $.5(.4-.6)$ & $.4(.3-.5)$ & $.3(.3-.4)$ & $.4(.3-.4)$ & $.4(.3-.4)$ & $.4(.3-.4)$ & $.3(.3-.4)$ & $.3(.2-.3)$ \\
\hline 4 & $.3(.2-.6)$ & $.3(.2-.4)$ & $.2(.1-.4)$ & $.2(.1-.3)$ & $.2(.1-.3)$ & $.2(.1-.2)$ & $.1(.1-.2)$ & $.1(.1-.1)$ \\
\hline
\end{tabular}

Values are presented as OR $(95 \% \mathrm{CI})$.

$\mathrm{CI}=$ confidence interval; $\mathrm{OR}=$ odds ratio.

a Adolescents in the same time period living with both parents, with at least one parent with higher than basic education, and both parents employed is used as a reference category. 
changes were observed among adolescents with most socioeconomic adversities. The relative differences in frequent smoking also increased over the study period.

The association between adolescent smoking and parental education has been observed in earlier studies [7,8]. Parents with low education level are more likely to smoke [30,31], and parental smoking is a major risk factor of adolescent smoking [32]. Parents with higher education may also know more about the adverse health effects of smoking and thus have more disapproval of the substance. The association between adolescent smoking and not living with both parents also corroborates earlier studies [18-20]. Children of divorced parents experience on average more stressful life events and have more mental health problems than children of nondivorced parents, which predispose adolescents to smoking [33,34]. To the best of our knowledge, the association between adolescent smoking and parental unemployment has not been studied previously. Parental unemployment is associated with financial problems in the family and adolescent psychosocial problems, which are known risk factors of adolescent smoking [35,36].

Most importantly, although the overall proportion of frequent smoking decreased from 2000 to 2015, no similar decrease was observed among adolescents with most socioeconomic adversities. This resulted in an increase in relative socioeconomic differences across the study years. Similarly, although the overall prevalence of lifelong nonsmoking increased, no similar increase was observed among adolescents with most socioeconomic adversities. However, relative differences in lifelong nonsmoking varied only slightly over time. Increased socioeconomic disparities in adolescent smoking have also been observed in other studies over Europe in the 21st century [9,25,27]. Smoking prevention programs have been shown to be less effective in lower socioeconomic groups [37], which may partly explain why smoking has not decreased in lower socioeconomic groups despite strong national tobacco policy. Therefore, new preventive efforts targeted at adolescents with socioeconomic adversities should be considered. It is also possible that hardship in the lowest socioeconomic groups has increased over time. Societal changes, such as increases in long-term unemployment and decreases in social security benefits may have widened the gap between socioeconomic groups in the 21st century [38]. Decreasing socioeconomic health disparities is an important public health objective, as socioeconomic health disparities increase individual suffering and inflict burden on public health care and economy [28].

\section{Methodological considerations}

This study has several strengths; it is based on a nationwide population-based time trend study with a large sample size consisting of Finnish eighth and ninth graders $(n=761,278)$ and a high participation rate $(43 \%-82 \%$ of the whole age cohort of the country). The school sample of this age group is comprehensive as basic education is compulsory for everyone under the age of 16 years in Finland. The measurement of smoking, sampling, and timing of the study were held constant over the study years. This study addressed both absolute and relative socioeconomic differences, which are both important when studying changes in socioeconomic disparities over time [39].

This study has also some limitations. Self-report data are susceptible to errors, such as recall bias and mischievous responding. Especially parental education can be difficult for an adolescent to recall, which may have caused the proportion of missing responses on that question to be higher than on other questions. However, the proportions of missing responses on all questions studied were very small and thus did not affect the results. Mischievous responding is another source of error in studies relying on self-report data. Mischievous responders are defined as "youths who provide extreme, and potentially untruthful, responses to multiple questions" [40]. The degree of mischievous responding was not assessed in this study. However, there is no reason to assume that mischievous responding had changed over time.

The socioeconomic differences in adolescent smoking increased in Finland between years 2000 and 2015. Although the overall proportion of frequent smoking decreased over the study period, no similar decrease was observed among adolescents with the most socioeconomic adversities. Similarly, although the overall prevalence of lifelong nonsmoking increased, this was not observed among adolescents with most socioeconomic adversities. Socioeconomic adversities should be considered in the prevention of adolescent smoking.

\section{Funding Source}

This research did not receive any specific grant from funding agencies in the public, commercial, or not-for-profit sectors.

\section{References}

[1] Saha SP, Bhalla DK, Whayne TF, et al. Cigarette smoke and adverse health effects: An overview of research trends and future needs. Int J Angiol 2007; 16:77-83.

[2] Das SK. Harmful health effects of cigarette smoking. Mol Cell Biochem 2003;253:159-65.

[3] WHO. Tobacco use in adolescence. Available at: http://users.jyu.fi/ jtynjala/press/WHO-HBSC_factsheet_Tobacco_AW2.pdf. Accessed December 29, 2017.

[4] Wallace JM, Vaughn MG, Bachman JG, et al. Race/ethnicity, socioeconomic factors, and smoking among early adolescent girls in the United States. Drug Alcohol Depend 2009;104:S42-9.

[5] Gadalla TM. Unhealthy behaviours among Canadian adolescents: Prevalence, trends and correlates. Chronic Dis Inj Can 2012;32:156-63.

[6] Gielkens-Sijstermans CM, Mommers MA, Hoogenveen RT, et al. Reduction of smoking in Dutch adolescents over the past decade and its health gains: A repeated cross-sectional study. Eur J Public Health 2010;20:146-50.

[7] Kuntz B, Lampert T. Smoking and passive smoke exposure among adolescents in Germany. Dtsch Arztebl Int 2016;113:23-30.

[8] Moberg DP, Rettammel RJ. Tobacco use trends and correlates among students in the Madison metropolitan school district. Wis Med J 2001;100: $43-8$.

[9] Rasmussen M, Due P, Damsgaard MT, et al. Social inequality in adolescent daily smoking: Has it changed over time? Scand J Public Health 2009;37: 287-94.

[10] Finnish tobacco control policy and legislation. Available at: https://thl.fi/fi/ web/alcohol-tobacco-and-addictions/tobacco/finnish-tobacco-control-policyand-legislation. Accessed October 22, 2018.

[11] Tobacco Act. Available at: https://savutonsuomi.fi/en/towards-tobaccofree-finland/tobacco-act/. Accessed October 23, 2018.

[12] Okoli C, Greaves L, Fagyas V. Sex differences in smoking initiation among children and adolescents. Public Health 2013;127:3-10.

[13] Selya AS, Dierker LC, Rose JS, et al. Risk factors for adolescent smoking: Parental smoking and the mediating role of nicotine dependence. Drug Alcohol Depend 2012;124:311-8.

[14] Pampel FC, Boardman JD, Daw J, et al. Life events, genetic susceptibility, and smoking among adolescents. Soc Sci Res 2015;54:221-32.

[15] Audrain-McGovern J, Rodriguez D, Kassel JD. Adolescent smoking and depression: Evidence for self-medication and peer smoking mediation. Addiction 2009;104:1743-56.

[16] Krieger N, Williams DR, Moss NE. Measuring social class in US public health research: Concepts, methodologies, and guidelines. Annu Rev Public Health 1997; 18:341-78.

[17] Galobardes B, Shaw M, Lawlor DA, et al. Indicators of socioeconomic position (part 1). J Epidemiol Community Health 2006;60:7-12. 
[18] Wallace JM, Forman TA, Guthrie BJ, et al. The epidemiology of alcohol, tobacco and other drug use among black youth. J Stud Alcohol 1999;60: $800-9$.

[19] Ledoux S, Miller P, Choquet M, et al. Family structure, parent-child relationships, and alcohol and other drug use among teenagers in France and the United Kingdom. Alcohol Alcohol 2002;37:52-60.

[20] Du Y, Palmer PH, Sakuma K-L, et al. The association between family structure and adolescent smoking among multicultural students in Hawaii. Prev Med Rep 2015;2:206-12.

[21] Rogers EM. Diffusion of innovations. 1995.

[22] Lopez AD, Collishaw NE, Piha T. A descriptive model of the cigarette epidemic in developed countries. Tob Control 1994;3:242-7.

[23] Huisman M, Kunst AE, Mackenbach JP. Educational inequalities in smoking among men and women aged 16 years and older in 11 European countries. Tob Control 2005;14:106-13.

[24] van Zon SKR, Bültmann U, Mendes de Leon CF, et al. Absolute and relative socioeconomic health inequalities across age groups. PLoS One 2015;10: e0145947.

[25] de Looze M, ter Bogt T, Hublet A, et al. Trends in educational differences in adolescent daily smoking across Europe, 2002-10. Eur J Public Health 2013; 23:846-52.

[26] Richter M, Leppin A. Trends in socio-economic differences in tobacco smoking among German school children. Eur J Public Health 2007;17: $565-71$.

[27] Doku D, Koivusilta L, Rainio S, et al. Socioeconomic differences in smoking among Finnish adolescents from 1977 to 2007. J Adolesc Health 2010;47: $479-87$.

[28] Koskinen S, Martelin T. Current public health problems and their prevention. In: Restraining Healthcare Expenditures: The Significance of Financial System and Prevention. Helsinki: Prime Minister’s Office; 2007.
[29] Knaappila N, Marttunen M, Fröjd S, et al. Socioeconomic trends in school bullying among Finnish adolescents from 2000 to 2015. Child Abuse Negl 2018;86:100-8

[30] Gilman SE, Martin LT, Abrams DB, et al. Educational attainment and cigarette smoking: A causal association? Int J Epidemiol 2008;37:615-24.

[31] Dobson R. Poor more likely to smoke and less likely to quit. BMJ 2004;328: 914.

[32] Gilman SE, Rende R, Boergers J, et al. Parental smoking and adolescent smoking initiation: An intergenerational perspective on tobacco control, Pediatrics 2009;123:e274-81.

[33] Amato P, Keith B. Parental divorce and the well-being of children: A metaanalysis. Psychol Bull 1991;110:26-46.

[34] Rajesh V, Diamond PM, Spitz MR, et al. Smoking initiation among Mexican heritage youth and the roles of family cohesion and conflict. J Adolesc Health 2015;57:24-30.

[35] Kim Y, Hagquist C. Trends in adolescent mental health during economic upturns and downturns: A multilevel analysis of Swedish data 1988-2008. J Epidemiol Community Health 2017;72:101-8.

[36] Baum A, Fleming R, Reddy DM. Unemployment stress: Loss of control, reactance and learned helplessness. Soc Sci Med 1986:22:509-16.

[37] Pampel FC. Diffusion, cohort change, and social patterns of smoking. Soc Sci Res 2005;34:117-39.

[38] Moisio P. Minimum social security benefit and poverty line in Finland report for comprehensive social security reform (Sata) committee. Helsinki: Openings; 2009.

[39] Houweling TA, Kunst AE, Huisman M, et al. Using relative and absolute measures for monitoring health inequalities: Experiences from cross-national analyses on maternal and child health. Int J Equity Health 2007;6:15.

[40] Robinson-Cimpian JP. Inaccurate estimation of disparities due to mischievous responders. Educ Res 2014;43:171-85. 